$\underline{\text { Preprint typeset in JHEP style - HYPER VERSION }}$

KIAS P11035

HIP-2011-18/TH

\title{
Production of two Higgses at the Large Hadron Collider in CP-violating MSSM
}

\author{
Priyotosh Bandyopadhyay $^{a, 1}$ and Katri Huitu ${ }^{a, 2}$ \\ ${ }^{a}$ Department of Physics, and Helsinki Institute of Physics, \\ P.O.Box 64 (Gustaf Hällströmin katu 2), FIN-00014 University of Helsinki, Finland \\ Email: priyotosh.bandyopadhyay@helsinki.fi, ${ }^{2}$ katri.huitu@helsinki.fi
}

\begin{abstract}
Production of two Higgs bosons is studied in a $\mathrm{CP}$ violating supersymmetric scenario at the Large Hadron Collider with $E_{c m}=14 \mathrm{TeV}$. There exists a region where a very light Higgs $\lesssim 50 \mathrm{GeV}$ could not be probed by LEP experiment. This leads to so called 'LEP hole' region. Recently LHC found a Higgs boson around $\sim 125 \mathrm{GeV}$, which severely constrains the possibility of having lighter Higgs bosons, which cannot be detected, i.e., buried Higgs, in this model. We investigate the possibility of buried Higgs bosons along with the direct and indirect bounds coming from LEP, LHC and other experiments. In particular we take into account the constraints from EDM and from $B$-observables. We analyse first the case where a Higgs boson mass is around $125 \mathrm{GeV}$ and the other two Higgs masses are below $100 \mathrm{GeV}$ and not observabed so far. In the second case the lightest Higgs boson mass is around $125 \mathrm{GeV}$ and the other two are decoupled. We analyse the production of two Higgses and their decay modes leading to various final states for these benchmark points. We perform a collider simulation with PYTHIA and Fastjet where we consider all the major backgrounds. Among the final states we have analysed, we find that $2 b+2 \tau$ is promising and the signal significance is $5 \sigma$ at an integrated luminosity $\lesssim 10 \mathrm{fb}^{-1}$. For some benchmark points it is also possible to observe the light Higgs mass peak. We also explore the leptonic final state which could be instrumental in the precision measurement of a very light Higgs.
\end{abstract}

Keywords: Higgs, CP-violation, Supersymmetry, LHC. 


\section{Contents}

1. Introduction 1

2. CP violating scenario and the experimental constraints 3

3. Benchmark points for collider study

4. Collider phenomenology 6

4.1 Sig1: $3 b+2 \tau$

4.2 Sig2: $2 b+2 \tau$

4.3 Sig3: $2 \ell \quad 13$

5. Summary and discussion 14

\section{Introduction}

$\mathrm{CP}$ violation is among the phenomena which are not fully understood in the context of the Standard Model (SM). Although CP violation exists in the SM, and agrees well with the laboratory experiments, there is an inconsistency between the amount of violation and matter content of the Universe, and it is argued that new sources of $\mathrm{CP}$ violation are needed.

Many of the proposals for beyond the SM (BSM) physics do contain new sources for $\mathrm{CP}$ violation. In this work we consider Minimal Supersymmetric Standard Model (MSSM). It has been shown in the literature that the tree-level CP invariance of the MSSM Higgs potential can be violated by loop effects involving CP-violating interactions of Higgs bosons to top and bottom squarks [1, 2, 3, 4, 5, 6]. In such a scenario with explicit CP-violation at tree-level, the neutral Higgses $\left(h_{i}, \mathrm{i}=1,2,3\right)$ mix the CP states at loop-level. It has been shown that [2, 4, 5] loop-induced CP-violation modifies the tree-level Higgs coupling such that light Higgs in this scenario could be $\lesssim 60 \mathrm{GeV}$ and can escape the detection at LEP2.

For example, it has been shown that assuming universality of gaugino masses $\left(M_{i}\right.$, $\mathrm{i}=1,2,3)$ at some high scale and assuming corrections from third generation strong sector, the CP-violating MSSM Higgs sector can be parametrised in terms of a few independent phases [7]: the phase of Higgsino mass parameter (also called $\mu$ term), i.e., $\operatorname{Arg}(\mu)$, and the phase of soft trilinear supersymmetry (SUSY) breaking parameters, i.e., $\operatorname{Arg}\left(A_{f}\right)$, with $f=t, b$. The experimental upper bounds on the electric dipole moments (EDMs) of electrons and neutrons [8, 9] as well as of mercury atoms [10] constrain these phases.

Before Large Hadron Collider (LHC) found out a Higgs resonance with mass around $125 \mathrm{GeV}$ 11, 12, 13, 14] earlier colliders had given bounds on the Higgs mass. For the SM 
Higgs boson, mass bound from the LEP collider is $m_{h}>114.4 \mathrm{GeV}$ [15, 16], and Tevatron excludes Higgs for the mass ranges $m_{h} \sim 147-180 \mathrm{GeV}$ and $100-103 \mathrm{GeV}$ but finds an excess in 115-135 $\mathrm{GeV}$ region [17]. In the MSSM with real and $\mathrm{CP}$-conserving parameters, the experimental lower limit on the lightest Higgs boson is $\sim 90 \mathrm{GeV}$ [18 for any $\tan \beta$. The lower bound on the mass of the lightest Higgs boson of the CP-conserving MSSM from LEP [16] can be drastically reduced or may even entirely vanish if non-zero CP-violating phases are allowed [19, 20]. This can happen through radiative corrections to the Higgs potential, whereby the above mentioned phases of the $\mu$ parameter and the $A$ parameters enter into the picture [1, 21].

With the discovery of $\sim 125 \mathrm{GeV}$ Higgs at the LHC the question of a buried Higgs remains to be answered. The LHC experiment will look in all possible different decay modes to find an extra scalar which would be lighter than $100 \mathrm{GeV}$. Finding of such scalar(s) will be certainly a proof of BSM Higgs but also the possibility of CP-violating MSSM will come into the picture. The phenomenology of such a light Higgs has been studied in the context of CPV-MSSM in a benchmark scenario known as 'CPX' [1, 21].

In the CPX scenario the $Z Z h_{1}$ coupling can be strongly reduced because of the CP violating phases, and the LEP mass limit for the lightest Higgs boson can be lowered to 50 $\mathrm{GeV}$ or even less, depending on $\tan \beta$. Thus the LEP searches leave a hole in $\left(m_{h_{1}}, \tan \beta\right)$ parameter space [16]. Complementary channels such as $e^{+} e^{-} \rightarrow h_{1} h_{2}$ suffer also phase space suppression within the hole region. At Tevatron, this CP violation and the Higgs phenomenology has been studied [22, 23].

Within the hole region in addition to $Z Z h_{1}$ coupling, $W W h_{1}$ and $t t h_{1}$ are suppressed and thus the lightest Higgs boson $h_{1}$ is difficult to discover. In the context of CPX scenarios there has been quite a few studies performed in the SM production channels [24] as well as in the supersymmetric channels [25, 26, 27]. In the context of CP-conserving MSSM, cascade Higgs production has been studied in [28].

Most of these earlier studies do not fit with the data for $\sim 125 \mathrm{GeV}$ Higgs and the other experimental constraints coming from EDMs and the rare $B$-decays. In this article we consider the recent SUSY mass bounds from LHC along with the Higgs results. We take into account thallium EDM result and constraints coming from $\operatorname{Br}\left(B_{s} \rightarrow s \gamma\right)$ and $\operatorname{Br}\left(B_{s} \rightarrow \mu \mu\right)$. We look for the possibility of the buried Higgs or the decoupled Higgs scenarios as two possibilities. In this context we study the Higgs pair production. We consider the $H \rightarrow b \bar{b}, \tau \bar{\tau}, \ell \bar{\ell}$ decay modes for possible final states. We find that $2 b+2 \tau$ channel is very promising in searching for a very light Higgs $\left(m_{h_{1}} \sim 30 \mathrm{GeV}\right)$ and $\lesssim 10 \mathrm{fb}^{-1}$ of integrated luminosity will be enough to have $5 \sigma$ significance over the dominant $\mathrm{SM}$ backgrounds. For the precision measurement leptonic channel would be crucial. We also find out that Higgs productions in association with $Z$ will also contribute to these final states. One can differentiate between the two types of contributions by constructing the heavier Higgs mass peak, i.e., $m_{h_{2,3}}$ in the corresponding channels at very high luminosity.

We will also point out that in certain benchmark points the two Higgs production through coupling of three Higgs bosons is important, and thus we have a possibility to probe the Higgs potential at those points. Obviously construction of the Higgs potential would be of fundamental importance. 
The paper is organised as follows. In Section 2 we review the CPX benchmark scenario and discuss the experimental constrains. We also discuss very briefly the possibilities of evading such bounds. In section 3 we define the benchmark points consistent with the experimental results for the collider study. The corresponding production cross-section and the decay branching fractions are listed in this section. In section 4 we carry out collider simulation for $14 \mathrm{TeV}$ LHC for the desired final states. Finally in section 5 we summarise.

\section{CP violating scenario and the experimental constraints}

It is known [1, 21] that the $C P$-mixing term in the Higgs sector is generated at quantum level and proportional to $\operatorname{Im}\left(\mu A_{t}\right) / M_{S U S Y}^{2}$. The well known CPX scenario predicts that certain parameters are related:

$$
\begin{aligned}
& m_{\tilde{t}}=m_{\tilde{b}}=m_{\tilde{\tau}}=M_{S U S Y},\left|A_{t}\right|=\left|A_{b}\right|=\left|A_{\tau}\right|=2 M_{S U S Y}, \\
& \arg \left(A_{t}\right)=\arg \left(A_{b}\right)=\arg \left(A_{\tau}\right)=90^{0} .
\end{aligned}
$$

In particular the parameter space with $M_{S U S Y}=500 \mathrm{GeV}$ is of special phenomenological interest along with the other parameters that are compatible with the LEP "hole" and are given below,

$$
\begin{aligned}
& M_{S U S Y}=500 \mathrm{GeV}, \quad\left|m_{\tilde{g}}\right|=1 \mathrm{TeV}, M_{2}=2 M_{1}=200 \mathrm{GeV}, \\
& \arg \left(A_{b, \tau}\right)=90^{\circ}, \quad \arg \left(m_{\tilde{g}}\right)=90^{\circ}, \tan \beta=5-10 .
\end{aligned}
$$

In addition, $\tan \beta$ and $m_{H^{ \pm}}$are the free input parameters that could be varied to achieve various points in the 'LEP hole'. The consequences of the CPX scenario have been studied in 29.

Recently new results from LHC have changed the scenario as most of the parameter region is ruled out. In this paper we shall take into account the Higgs discovery around $\sim 125 \mathrm{GeV}$ which has been reported by the CMS and ATLAS collaborations [11, 12]. Along with the recent LHC Higgs results we also consider the Higgs bounds from LEP [30]. We can see that buried Higgs, i.e., a very light Higgs $(\lesssim 60 \mathrm{GeV})$, is still possible in MSSM. The possibility of a light Higgs could be an artifact of explicit CP-violation in the Lagrangian and then a loop-induced CP-violation in the Higgs sector as explained in the introduction.

The recent studies on some indirect variables show that they can constrain these CPviolating phases and eventually can rule out a large amount of parameter space. The bounds on the CP-violating MSSM coming from various dipole-moment measurements have been studied in details [31]. In this paper we consider the constraints coming from electric-dipole moment (EDM) of thallium with the current $2 \sigma$ upper bound $\left|d_{T l}\right|<1.3 \times 10^{-24}$ e cm [33]. For this purpose we vary the relative angles between $M_{1}, M_{2}$ and also $\phi_{A_{t}}, \phi_{M_{3}}$; where we denote $\operatorname{Arg}\left(A_{f}\right)=\phi_{f}$ and $\operatorname{Arg}\left(M_{i}\right)=\phi_{M_{i}}$. In this region the one loop-SUSY contribution and light Higgs mediated two-loop contribution are comparable and tend to 
cancel each other. Thus it is possible to achieve the desired EDM bounds. Here we would like to mention that a very light Higgs $\left(m_{h_{1}} \lesssim 8 \mathrm{GeV}\right)$ is ruled out from bottomonium decay $\Upsilon(1 S) \rightarrow \gamma h_{1}$ [34.

We also look into the flavour constraints coming from the $B$-observables. For this purpose we first consider $\operatorname{Br}\left(B_{s} \rightarrow \mu \mu\right)$, which recently has come down by two orders of magnitude 35] can severely constrain this scenario. $\operatorname{Br}\left(B_{s} \rightarrow \mu \mu\right)$ grows large as $\tan \beta$ increases. For the cancellation we use GIM operative point mechanism [36]: we vary

$\rho=\frac{Q_{1,2}}{Q_{3}}$, the ratio of first two generation of the squark masses over the third generation squark masses. The cancellation happens when $\rho \sim 0.8-1.9$. This predicts very light first two generation masses for some cases. To evade such light mass bound coming from jets $+\not p_{T}$ at the LHC [37], LSP mass must be large which would make the jets rather soft.

Next we consider the bounds coming from $\operatorname{Br}\left(B_{s} \rightarrow X_{s} \gamma\right)$ [38]. Unlike $B_{s} \rightarrow \mu \mu$ case $\operatorname{Br}\left(B_{s} \rightarrow X_{s} \gamma\right)$ decreases as $\tan \beta$ increases. This is because the charged Higgs contribution is suppressed due to the threshold corrections at large $\tan \beta$ [39]. We also included recent bounds on third-generation squark masses and on LSP from $8 \mathrm{TeV}$ LHC [40]. To have light third-generation mass $\left(M_{\mathrm{SUSY}}\right)$, the LSP needs to be relatively heavy, i.e., around $300 \mathrm{GeV}$ [40]. We also choose $m_{3}=1.4 \mathrm{TeV}$ to satisfy recent gluino mass bound [37, 41]. For this choice of gluino mass we find that it is very difficult to get $m_{h_{3}} \gtrsim 124 \mathrm{GeV}$ by using CPsuperH [42 $]^{1}$. We vary $\tan \beta$ and $m_{H^{ \pm}}$as usual as we move to different points in the 'LEP hole'. The Higgs mass spectrum depends on the radiative correction which is very sensitive to top mass. The central value of $m_{t}$ has shifted frequently during the years. These shifts change the size of the hole, although the location remains almost the same. We use for the top mass $173.2 \pm 0.9 \mathrm{GeV}$ as referred by Tevatron [44].

\section{Benchmark points for collider study}

After above investigation we find three points in explicit CP-violating MSSM which are no longer so called "CPX" points but experimentally allowed ones. Allowed regions of the parameter space have very different but attractive phenomenological consequences. Table 1 describes the benchmark points that we consider for our collider study. We consider three different scenarios:

1. Two light Higgses are buried and have masses $<100 \mathrm{GeV}$ and mass of the heaviest one is around $125 \mathrm{GeV}$.

2. The lightest Higgs is very light $m_{h_{1}} \leq 30 \mathrm{GeV}$, so that $h_{3} \rightarrow h_{1} Z$ is allowed. The second lightest is also buried, $m_{h_{2}} \leq 100 \mathrm{GeV}$ and mass of the third one $125 \mathrm{GeV}$ as in the previous case.

3. This is a decoupled scenario where the heavier Higgses are decoupled with masses $\geq 500 \mathrm{GeV}$ and the lightest one has $m_{h_{1}} \sim 125 \mathrm{GeV}$.

\footnotetext{
${ }^{1}$ There is $\sim 2-3 \mathrm{GeV}$ uncertainty in Higgs mass calculated by CPsuperH and FeynHiggs 43]. For this paper we have used CPsuperH2.0 for the mass spectrum and the other observables.
} 


\begin{tabular}{||c|c|c|c|}
\hline \hline Parameters & BP1 & BP2 & BP3 \\
\hline \hline $\tan \beta$ & 30 & 30 & 20 \\
\hline$m_{H^{ \pm}}$ & 115 & 115 & 500 \\
\hline$\mu$ & 1400 & 2000 & 1000 \\
\hline$M_{1}$ & 300 & 300 & 300 \\
$\phi_{M_{1}}$ & 66 & 66 & 40 \\
\hline$M_{2}$ & 400 & 400 & 400 \\
$\phi_{M_{2}}$ & 0 & 0 & 0 \\
\hline$M_{3}$ & 1400 & 1400 & 1400 \\
$\phi_{M_{3}}$ & 61 & 61 & 60 \\
\hline$A_{t}$ & 1000 & 1000 & 1000 \\
$\phi_{A_{t}}$ & 60 & 60 & 60 \\
\hline$A_{b}$ & 11200 & 4200 & 1000 \\
$\phi_{A_{b}}$ & 35 & 35 & 90 \\
\hline$A_{\tau}$ & 14200 & 16100 & 1000 \\
$\phi_{A_{\tau}}$ & 90 & 90 & 90 \\
\hline$\rho$ & 0.83 & 0.88 & 1.90 \\
\hline \hline$m_{h_{1}}$ & 54.25 & 25.00 & 123.50 \\
\hline$m_{h_{2}}$ & 95.00 & 94.70 & 490.70 \\
\hline$m_{h_{3}}$ & 124.40 & 124.60 & 494.70 \\
\hline \hline
\end{tabular}

Table 1: Input parameters in the benchmark points within the 'LEP-hole' and the corresponding $\mathrm{CP}$-violating neutral Higgs masses. The angles are given in the unit of degree and other parameters are in $\mathrm{GeV}$ except $\tan \beta$ which is unitless.

\begin{tabular}{||c||c|c|c|c|c|c||}
\hline \multicolumn{1}{||c||}{ Benchmark } & \multicolumn{7}{c|}{ Cross-section in fb } \\
Points & $\sigma_{h_{1} h_{2}}$ & $\sigma_{h_{1} h_{3}}$ & $\sigma_{h_{1} h_{1}}$ & $\sigma_{h_{2} h_{2}}$ & $\sigma_{h_{3} h_{3}}$ & $\sigma_{h_{2} h_{3}}$ \\
\hline \hline BP1 & 908.02 & 47.02 & 5393.50 & 24.11 & 7.83 & 6.92 \\
\hline BP2 & 1858.89 & 45.23 & 33086.7 & 20.35 & 5.19 & 3.91 \\
\hline BP3 & $1.73 \times 10^{-2}$ & $1.0 \times 10^{-2}$ & 18.6 & $8.6 \times 10^{-3}$ & $5.7 \times 10^{-3}$ & 0.47 \\
\hline \hline
\end{tabular}

Table 2: Cross-sections (in fb) of two Higgs productions $\left(h_{2,3} h_{i}=1,2,3\right)$ at the LHC with $E_{c m}=$ $14 \mathrm{TeV}$ for the benchmark points.

In this study, we focus on two Higgs production processes, i.e., $h_{i} h_{j}, \mathrm{i}=1,2,3$ and $j=2,3$. We investigate the various possible decays of the Higgs bosons which will lead to the corresponding final states. We also include Higgs production in association with a $Z$ boson. Table 2 presents the cross-sections of two Higgs boson productions $\left(h_{i} h_{j}, i, j=1,2,3\right)$ for the three benchmark points at the $\mathrm{LHC}$ with $\mathrm{ECM}=14 \mathrm{TeV}$ and Table 3 presents the cross-sections of Higgs boson productions associated with a $Z$ boson for the center of mass energy of $14 \mathrm{TeV}$.

Table 1 and Table 5 present the decay branching fractions of $h_{1}, h_{2}$ and $h_{3}$, respectively. 


\begin{tabular}{||c||c|c|c|}
\hline \multicolumn{1}{||c||}{ Benchmark } & \multicolumn{3}{c|}{ Cross-section in fb } \\
Points & $\sigma_{h_{1} Z}$ & $\sigma_{h_{2} Z}$ & $\sigma_{h_{3} Z}$ \\
\hline \hline BP1 & 513.18 & 155.39 & 672.74 \\
\hline BP2 & 1180.31 & 150.248 & 672.93 \\
\hline BP3 & 708.56 & 59.00 & 53.86 \\
\hline \hline
\end{tabular}

Table 3: Cross-sections (in fb) of Higgs productions $\left(h_{2,3} h_{i}=1,2,3\right)$ associated with $Z$ boson at the LHC with $E_{c m}=14 \mathrm{TeV}$ for the benchmark points.

\begin{tabular}{||c||c|c|c|c||}
\hline \hline \multicolumn{1}{||c||}{ Benchmark } & \multicolumn{4}{c||}{$h_{1}$ decays } \\
\hline points & $b \bar{b}$ & $\tau \bar{\tau}$ & $W W$ & $Z Z$ \\
\hline \hline $\mathrm{BP} 1$ & 0.70 & 0.29 & - & - \\
\hline $\mathrm{BP} 2$ & 0.67 & 0.32 & - & - \\
\hline $\mathrm{BP} 3$ & 0.67 & 0.076 & 0.14 & 0.017 \\
\hline \hline
\end{tabular}

Table 4: The dominant branching fractions of the lightest Higgs boson $h_{1}$ for the benchmark points.

From Table 1 we can see that for all the three benchmark points the lightest Higgs, $h_{1}$ mostly decays to $\tau \tau$ and $b b$. In case of BP3, we have two additional decay modes $W W$ and $Z Z$. Similar to $h_{1}$, we can see from Table 5 that $h_{2}$ also mainly decays to $\tau \tau$ and $b b$. There is also a possibility to decay into $h_{1} Z$, the branching fractions of which are rather small. In case of BP2 and BP3 $h_{2} \rightarrow h_{1} h_{1}$ has small but non-zero branching fraction. Table 5 shows that the heaviest neutral Higgs $h_{3}$ mainly decays to $h_{1}$ pair for BP1 and BP2. In case of BP3 it decays to $\tau$ and $b$ pairs like $h_{1}$.

\section{Collider phenomenology}

In this study, CalcHEP [45] is used to calculate the cross-sections, the decay branching fractions and also to generate the events. The couplings and mass spectra are originally generated from the program CPsuperH2.2 [42] which is used by CalcHEP via calling the program CPsuperH2 2. The standard CalcHEP-PYTHIA interface 46], which uses the SLHA interface [47] was then used to pass the CalcHEP-generated events to PYTHIA [48]. Furthermore, all relevant decay information is generated with CalcHEP and is passed to PYTHIA through the same interface. All these are required since there is no public implementation of $\mathrm{CP}$ violating MSSM in PYTHIA. Subsequent decays of the produced particles, hadronization and the collider analyses are done with PYTHIA (version 6.4.5).

We use CTEQ6L parton distribution function (PDF) [49, 50]. In CalcHEP we opted for the lowest order $\alpha_{s}$ evaluation, which is appropriate for a lowest order PDF like CTEQ6L. 


\begin{tabular}{||c||c|c|c|c||c|c|c|c||}
\hline \hline \multicolumn{9}{|c||}{ Branching fraction } \\
\hline Benchmark & \multicolumn{9}{c||}{$h_{2}$ decays } & \multicolumn{4}{c||}{$h_{3}$ decays } \\
\hline points & $b \bar{b}$ & $\tau \bar{\tau}$ & $h_{1} Z$ & $h_{1} h_{1}$ & $b \bar{b}$ & $\tau \bar{\tau}$ & $h_{1} Z$ & $h_{1} h_{1}$ \\
\hline \hline BP1 & 0.68 & 0.316 & $1.0 \times 10^{-4}$ & - & 0.01 & $8.7 \times 10^{-3}$ & $1.6 \times 10^{-5}$ & 0.98 \\
\hline BP2 & 0.62 & 0.36 & $1.0 \times 10^{-3}$ & 0.01 & $6.7 \times 10^{-3}$ & $8.2 \times 10^{-3}$ & $3.4 \times 10^{-4}$ & 0.98 \\
\hline BP3 & 0.79 & 0.19 & $3.1 \times 10^{-4}$ & $1.4 \times 10^{-3}$ & 0.79 & 0.19 & $1.2 \times 10^{-4}$ & $1.9 \times 10^{-3}$ \\
\hline
\end{tabular}

Table 5: The dominant branching fractions of heavier Higgs bosons $\left(h_{2}\right.$ and $\left.h_{3}\right)$ for the benchmark points.

The renormalization/factorization scale in CalcHEP is set at $\sqrt{\hat{s}}$. This choice of scale results in a somewhat conservative estimate for the event rates.

For hadronic level simulation we have used Fastjet-3.0.3 [51] algorithm for the jet formation with the following criteria:

- the calorimeter coverage is $|\eta|<4.5$

- $p_{T, \text { min }}^{\text {jet }}=20 \mathrm{GeV}$ and jets are ordered in $p_{T}$

- leptons $(\ell=\mathrm{e}, \mu)$ are selected with $p_{T} \geq 20 \mathrm{GeV}$ and $|\eta| \leq 2.5$

- no jet should match with a hard lepton in the event

- $\Delta R_{l j} \geq 0.4$ and $\Delta R_{l l} \geq 0.2$

- Since efficient identification of the leptons is crucial for our study, we required, on top of the above set of cuts, that hadronic activity within a cone of $\Delta R=0.3$ between two isolated leptons should be $\leq 0.5 p_{T}^{\ell} \mathrm{GeV}$ in the specified cone.

In the CP-violating scenario, $h_{1}$ decays dominantly into $b \bar{b}$ and $\tau \bar{\tau}$ (see Table 4 ) for all the benchmark points as discussed in the earlier section. In cases of BP1 and BP2 where the light Higgs $h_{1}$ is relatively light $(<60 \mathrm{GeV}), b$-quarks lead to soft jets and the $b$-tagging efficiency is small. To illustrate this, we present in Figure 11 the ordered $p_{T}$ distributions for $b$-jets coming from $h_{1} h_{3}$ for BP1 and from $h_{1} h_{1}$ for BP3. We see that for BP1, the lowest $p_{T} b$-jet can be very soft, $p_{T} \leq 40 \mathrm{GeV}$. For this analysis we have required a $b$-jet tagging efficiency $(\geq 50 \%)$ 52].

Next we study the jet-multiplicity distribution for $h_{1} h_{3}$ for BP1, BP2 and the dominant background $t \bar{t}$. We can see from Figure 2 that the two Higgs production has fewer jets than the $t \bar{t}$. Demanding $n_{\text {jets }} \leq 4$ removes most of the $t \bar{t}$ background events. Thus it could be a very useful tool to kill the SM background as well as the SUSY cascade backgrounds which usually have a large number of jets. 


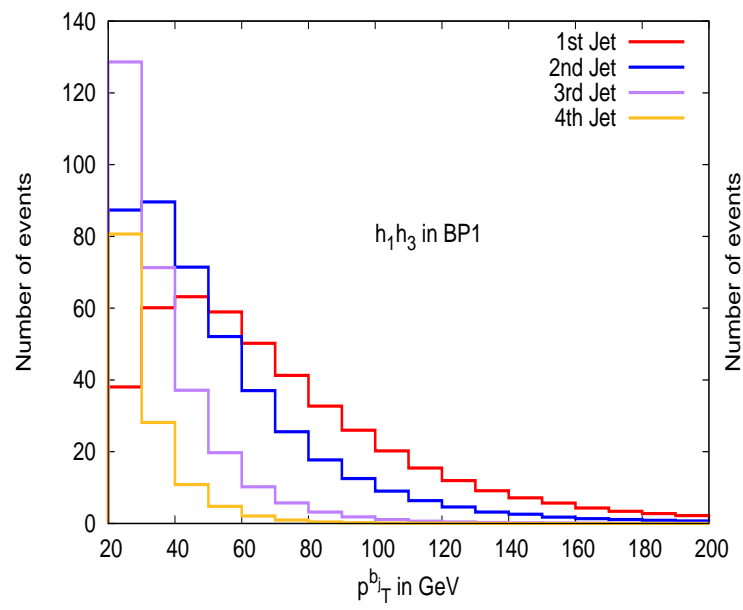

(a)

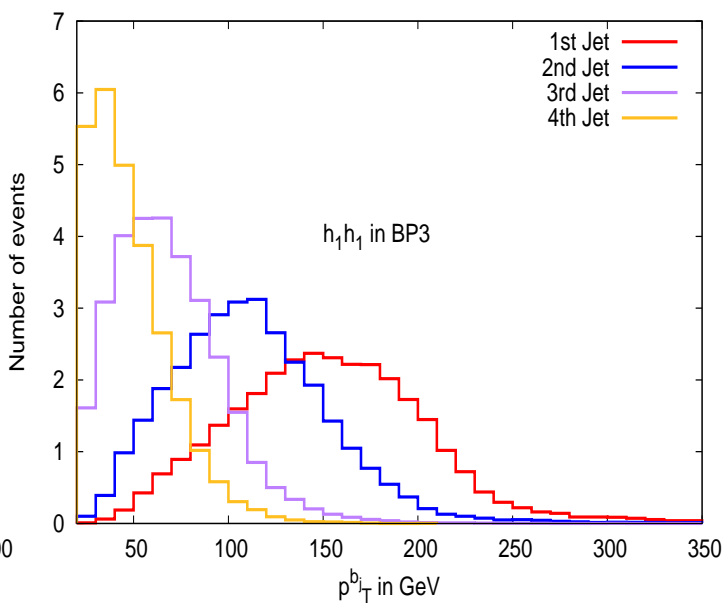

(b)

Figure 1: $p_{T}^{b_{j e t}}$ distribution for $h_{1} h_{3}$ (a) for BP1 and for $h_{1} h_{1}$ (b) for BP3 at an integrated luminosity of $\mathcal{L}=10 \mathrm{fb}^{-1}$.

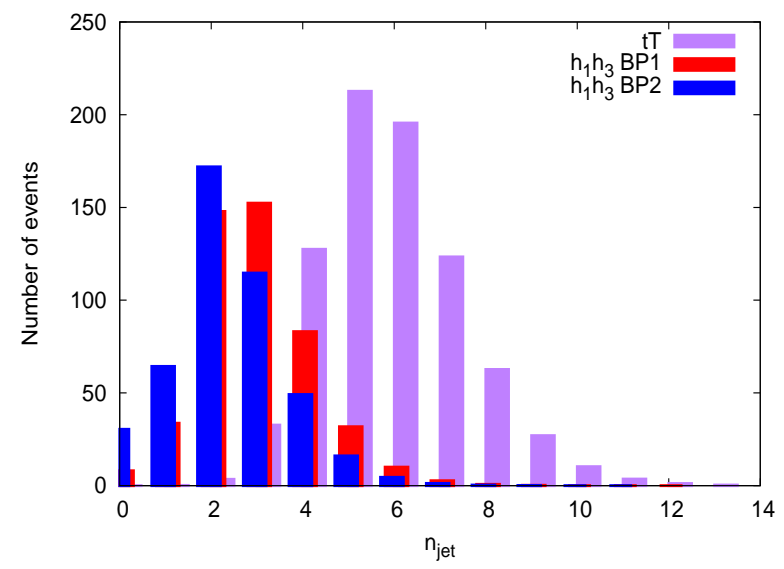

Figure 2: Jet multiplicity distributions for $h_{1} h_{3}$ for BP1, BP2 and $t \bar{t}$ at an integrated luminosity of $\mathcal{L}=10 \mathrm{fb}^{-1}$.

To see the status of the $b$-final states we first check the $b$-jet invariant mass. Figure 3 shows the invariant mass of two $b$-jets which satisfy the above mentioned criteria at an integrated luminosity of $10 \mathrm{fb}^{-1}$ at the LHC with center of mass energy of $14 \mathrm{TeV}$. In Figure 3 (a) the $b$-jet invariant mass comes from $h_{1} h_{1}$ signal for the three benchmark points. The lightest Higgs boson peaks are visible for all three benchmark points. In Figure 3(b) we show both the lightest Higgs $h_{1}$ peak as well as the second lightest Higgs peak $h_{2}$, which come from $h_{1} h_{2}$ for $\mathrm{BP} 1$ and $\mathrm{BP} 2^{2}$. Similarly Figure 3(c) describe the $b$-jet pair invariant mass distribution for BP1 and BP2 coming from $h_{1} h_{3}$ signal. Due to small cross-section the mass resolutions are not clear unlike the other two production channels.

\footnotetext{
${ }^{2}$ For BP3 the number of events are not enough to plot the $b$-jet pair invariant mass distribution
} 


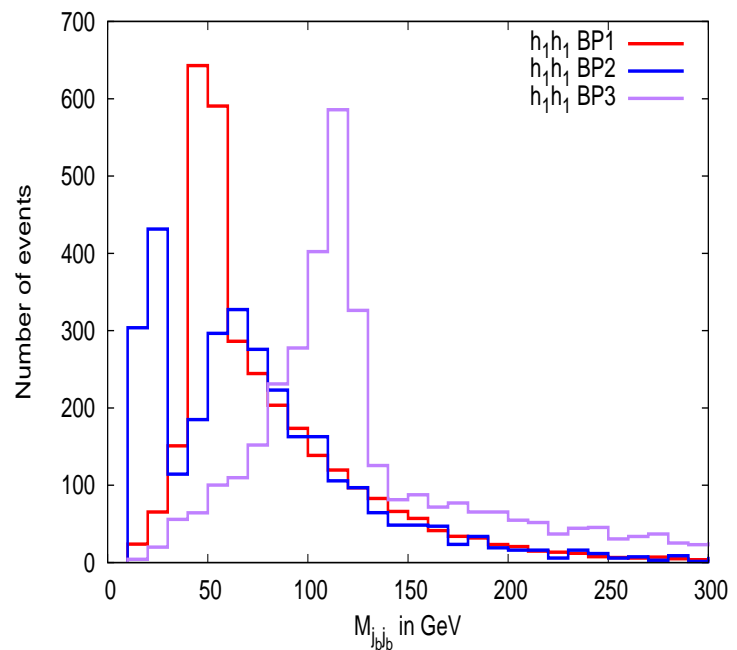

(a)

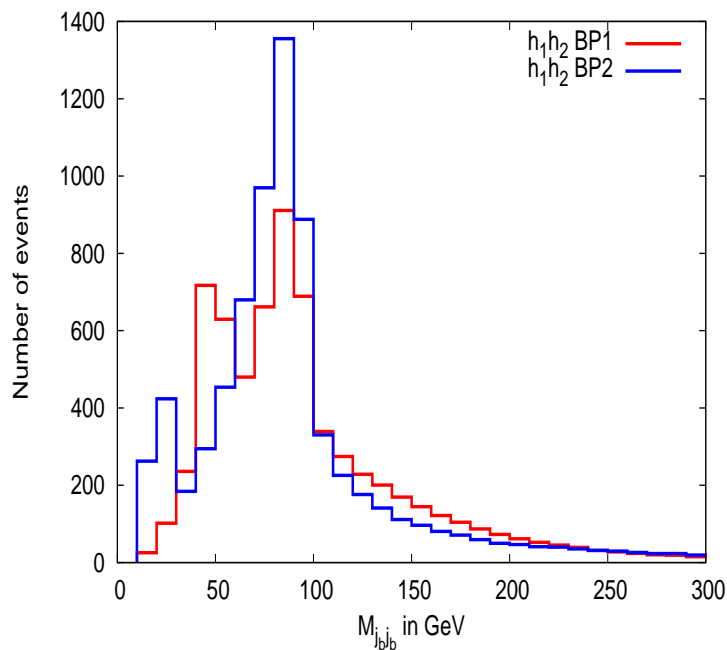

(b)

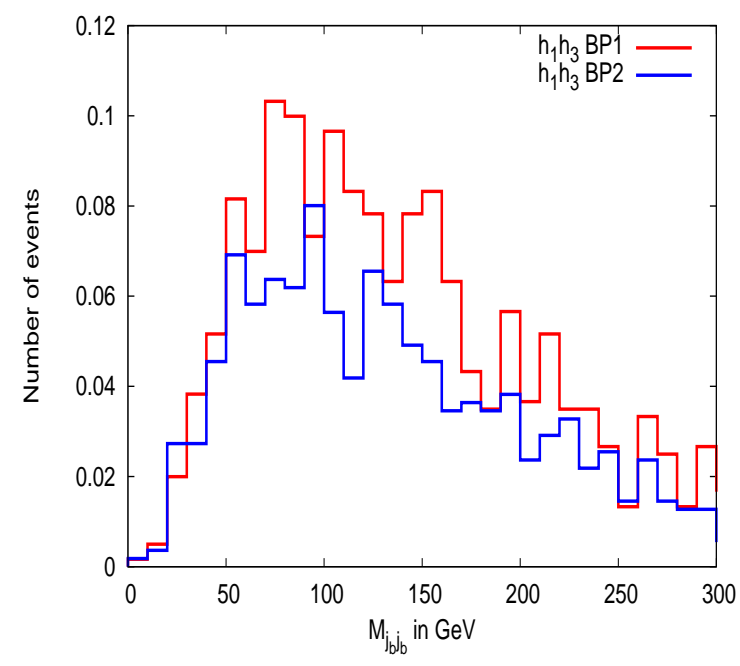

(c)

Figure 3: $b$-jet invariant mass distribution coming (a) from $h_{1} h_{1}$, (b) from $h_{1} h_{2}$ and (c) from $h_{1} h_{3}$ for benchmark points at an integrated luminosity of $\mathcal{L}=10 \mathrm{fb}^{-1}$.

All three Higgs bosons decay to $\tau$ pairs with branching fraction $\sim 8-30 \%$ except for $h_{3}$ whose branching fraction to $\tau$ pairs is $\mathcal{O}\left(10^{-3}\right)$ for BP1 and BP2 (see Table 4 and Table 5). For a very light Higgs, in cases of BP1 and BP2, the taus coming from $h_{1}$ can be very soft. Boost of the light Higgs $\left(h_{1}\right)$ of course increases the $p_{T}$ of taus. Figure $⿴$ (a) shows the $p_{T}$ distribution of the partonic $\tau$ coming from $h_{1} h_{1}$ production channel. We can clearly see $\tau$ s coming from $h_{1}$ decay for BP1 and BP2 will have enough boost to tag them as tau-jet. Figure 4 (b) shows that in case of $h_{1} h_{3}$ production channel the boost of $\tau \mathrm{s}$ increases further.

Taus coming from Higgs then decay to pions through one prong or/and three prong decay. In the present study, we use the one-prong (one charged track) hadronic decays 


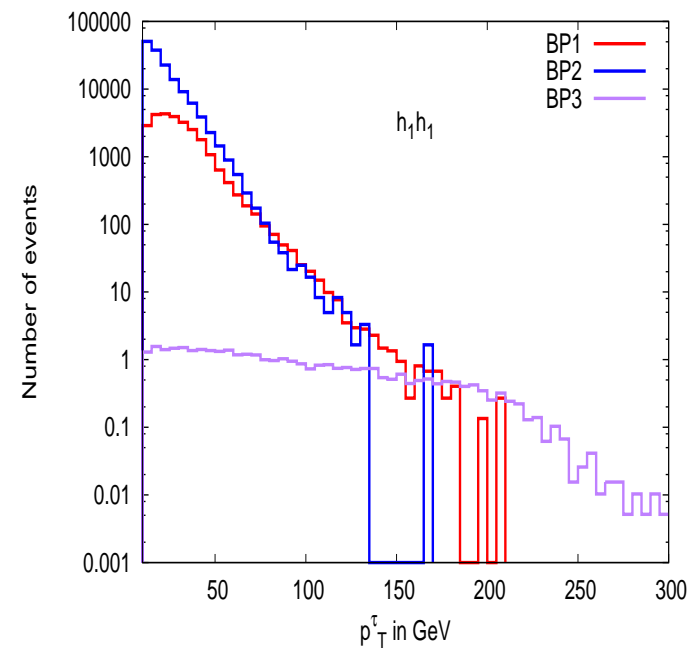

(a)

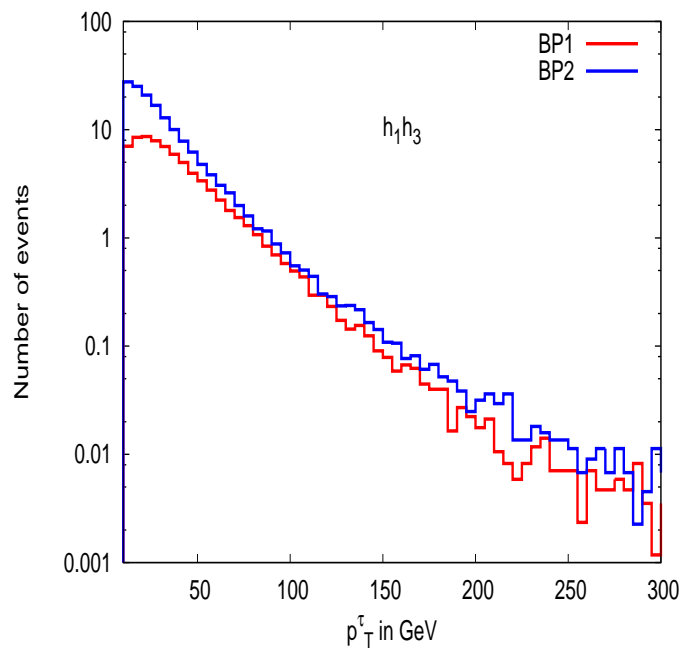

(b)

Figure 4: $p_{T}$ distribution of partonic $\tau$ coming from $h_{1} h_{1}$ and $h_{1} h_{3}$ for the benchmark points at an integrated luminosity of $\mathcal{L}=10 \mathrm{fb}^{-1}$. Due to small production cross-section of $h_{1} h_{3}$ for BP3, the generated events are not enough for the distribution.

of the $\tau$-leptons which have a collective branching fraction of about $50 \%$ of which almost $90 \%$ is comprised of final states with $\pi^{ \pm}, \rho$ and $a_{1}$ mesons. To establish a jet as a $\tau$-jet we take the following approach. We first check, for each jet coming out of Fastjet within $|\eta| \leq 2.5$, if there is a partonic $\tau$ within a cone of $\Delta R \leq 0.4$ about the jet-axis. If there is one, then we further ensure that there is a single charged track within a cone of $\Delta R \leq 0.1$ of the same jet axis. This marks a narrow jet character of a $\tau$-jet. Of course there is an efficiency associated to such kind of a geometric requirement which is a function of $p_{T}$ of the concerned jet and has been demonstrated in the literature [53, 54].

Next we study the the $\tau$ final state by plotting the hadronic $\tau$-jet invariant mass. In Figure 5 we plot the invariant mass distribution of two hadronic $\tau$-jets coming from the Higgs boson decay. In Figure 5(a) the contribution comes from $h_{1} h_{1}$ production as before and it is easily seen that the lightest Higgs mass peaks are much clearer than the $b$-jet invariant mass distributions. Figure $5(\mathrm{~b}) \&$ (c) show the contribution coming from the production of $h_{1} h_{2}$ and $h_{1} h_{3}$, respectively for BP1 and BP2. In case of Figure 5 (c) the $h_{3}$ mass peak is not visible as $h_{3}$ mostly decays to $h_{1}$ pair for both BP1 and BP2 (see table 5).

So far we have seen that the final states with $b$ and $\tau$-jets could be prompted for all the benchmark points if we consider the production channels, i.e., $h_{i} h_{j}, i, j=1,2,3$. Let us first discuss the final states with $b$ and $\tau$.

\subsection{Sig1: $3 b+2 \tau$}

The final state $3 b+2 \tau$ is possible when at least one Higgs is heavy, i.e., $h_{2}$ or $h_{3}$, which decays to $Z h_{1}$ or $h_{1} h_{1}$. If at least one lightest Higgs $h_{1}$ decays to tau lepton pair and the other $h_{1}$ or $Z$ decays to $b$ pairs then we have $4 b+2 \tau$ final state. This scenarios is possible 


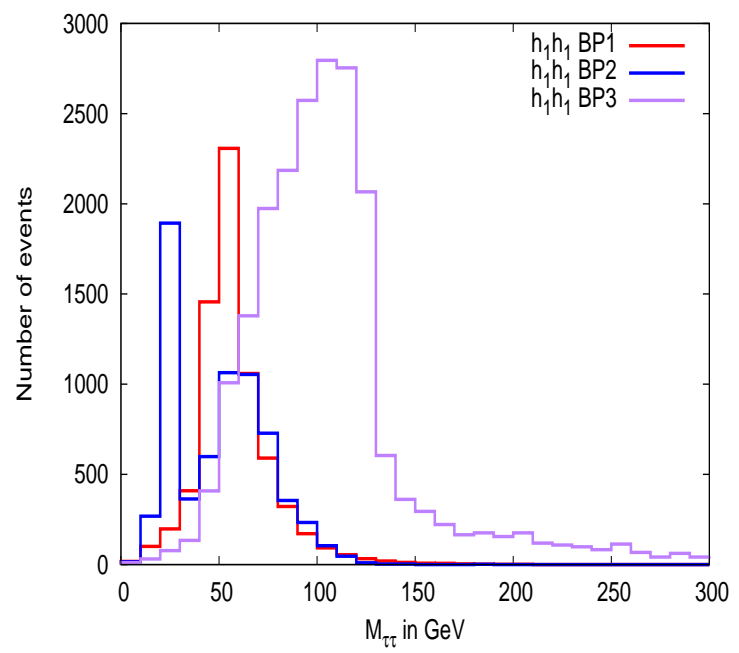

(a)

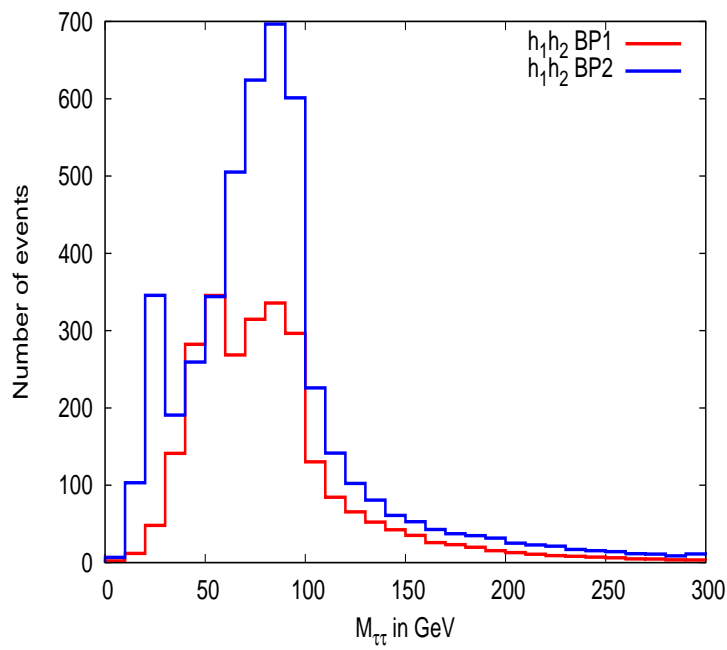

(b)

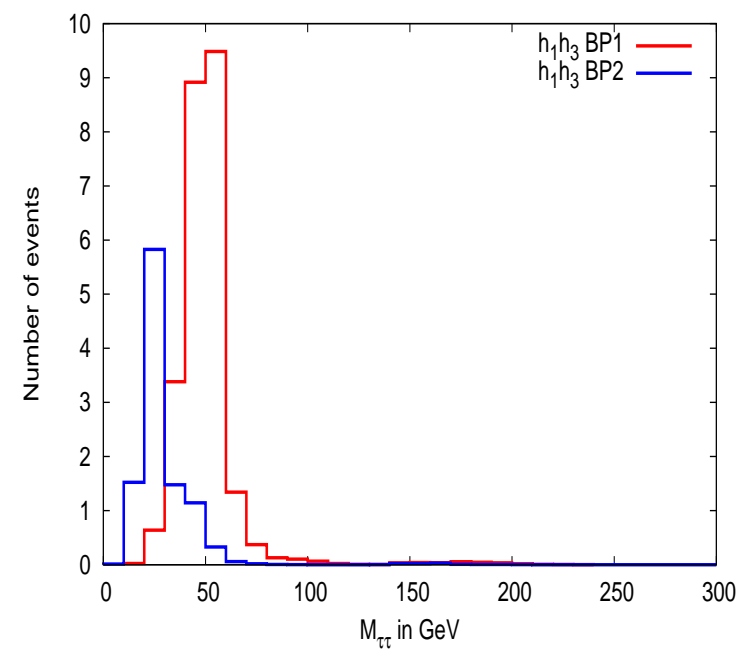

(c)

Figure 5: $\tau$-jet invariant mass distribution coming (a) from $h_{1} h_{1}$, (b) from $h_{1} h_{2}$ and (c) from $h_{1} h_{3}$ for benchmark points at an integrated luminosity of $\mathcal{L}=10 \mathrm{fb}^{-1}$.

for $\mathrm{BP} 1$ and $\mathrm{BP} 2$ :

$$
\begin{aligned}
p p & \rightarrow h_{1} h_{2,3}, \\
& \rightarrow h_{1} Z h_{1}\left(\text { or }_{1} \mathrm{~h}_{1}\right) \rightarrow 4 \mathrm{~b}+2 \tau .
\end{aligned}
$$

The $b$-tagging efficiency is around $50 \%$, so tagging $4 b$-jets will bring down the number of signal events. This is the reason we study the $3 b+2 \tau$ final state. We choose the final state as:

$$
\operatorname{sig} 1: \mathrm{n}_{\text {jets }} \leq 5+(\geq 3 \mathrm{~b}-\text { jet })+(\geq 2 \tau-\text { jet })+\left(p_{\mathrm{T}} \leq 30 \mathrm{GeV}\right)
$$


We consider $t \bar{t}, t \bar{t} Z, t \bar{t} W, Z Z$ and $t \bar{t} b \bar{b}$ as the main SM backgrounds. Table 6 presents the number of events for sig1 for signal and backgrounds at an integrated luminosity of 10 $\mathrm{fb}^{-1}$. Table 6 also presents the numbers of events with window cut of $\pm 10 \mathrm{GeV}$ around the mass peak of respective invariant mass distribution. Here we give the number of events with the window cuts around $h_{1}, h_{2}$ and $h_{3}$ for both $b b$ and $\tau \tau$ invariant mass distribution. Determination of these mass peaks depends on the relative number of signal events over background events.

\begin{tabular}{|c|c|c|c|c|c|c|c|c|}
\hline \multirow[t]{2}{*}{$\begin{array}{c}\text { Signal } \\
\text { Sign }\end{array}$} & \multicolumn{3}{|c|}{ Benchmark Points } & \multicolumn{5}{|c|}{ Backgrounds } \\
\hline & $\mathrm{BP} 1$ & $\mathrm{BP} 2$ & BP3 & $t \bar{t}$ & $t \bar{t} Z$ & $t \bar{t} W$ & $Z Z$ & $t \bar{t} b \bar{b}$ \\
\hline $\operatorname{sig} 1$ & 52.30 & 21.60 & 0.80 & 1.00 & 0.00 & 0.00 & 0.80 & 0.06 \\
\hline $\operatorname{sig} 1+\left|m_{b b}-m_{h_{1}}\right| \leq 10 \mathrm{GeV}$ & 25.30 & 8.80 & 0.20 & 0.00 & 0.00 & 0.00 & 0.00 & 0.00 \\
\hline $\operatorname{sig} 1+\left|m_{b b}-m_{h_{2}}\right| \leq 10 \mathrm{GeV}$ & 3.70 & 2.10 & 0.00 & 0.10 & 0.00 & 0.00 & 0.00 & 0.00 \\
\hline $\operatorname{sig} 1+\left|m_{b b}-m_{h_{3}}\right| \leq 10 \mathrm{GeV}$ & 1.80 & 0.12 & 0.00 & 0.00 & 0.00 & 0.00 & 0.00 & 0.00 \\
\hline $\operatorname{sig} 1+\left|m_{\tau \tau}-m_{h 1}\right| \leq 10 \mathrm{GeV}$ & 33.70 & 19.00 & 0.09 & 0.00 & 0.00 & 0.00 & 0.00 & 0.00 \\
\hline $\operatorname{sig} 1+\left|m_{\tau \tau}-m_{h 2}\right| \leq 10 \mathrm{GeV}$ & 33.70 & 19.00 & 0.09 & 0.00 & 0.00 & 0.00 & 0.60 & 0.00 \\
\hline $\operatorname{sig} 1+\left|m_{\tau \tau}-m_{h 3}\right| \leq 10 \mathrm{GeV}$ & 0.07 & 0.20 & 0.00 & 0.00 & 0.00 & 0.00 & 0.00 & 0.00 \\
\hline
\end{tabular}

Table 6: Number of events after the selection cuts for sig1 final states for the benchmark points and backgrounds at an integrated luminosity of $10 \mathrm{fb}^{-1}$ at the LHC with $E_{c m}=14 \mathrm{TeV}$.

From Table 6 we see that Sig1 has $7.1 \sigma$ significance over background for BP1 at an integrated luminosity of $10 \mathrm{fb}^{-1}$. For BP2 and BP3 it is $4.5 \sigma$ and $0.5 \sigma$, respectively. For $h_{1}$ peak we can get $\geq 5 \sigma$ significance for BP1 for both $b b$ and $\tau \tau$ invariant mass distribution. The corresponding numbers for BP2 are $3 \sigma$ and $4.4 \sigma$ respectively. In case of $h_{2}$ and $h_{3}$ peak, for a comparable signal significance over background one needs to go for higher luminosity.

Next we consider the case when two of the $h_{1}$ decay to tau pairs and the final state is $(\geq 2 b-j e t)+(\geq 4 \tau-j e t)$. The decay branching fraction of $h_{1} \rightarrow \tau \tau$ is around $30 \%$ which is much lower than the $h_{1} \rightarrow b \bar{b}$ branching fraction. The tau coming from such a light Higgs $\left(h_{1}\right)$ is of low $p_{T}$ which reduces the $\tau$ detection efficiency. Because of these two effects the final state does not have many events at $10 \mathrm{fb}^{-1}$ integrated luminosity.

There is a possibility that the heavier Higgses $\left(h_{2,3}\right)$ decay to $h_{1} Z$ in the case of $h_{1} h_{2,3}$ productions, which leads to $h_{1} h_{1} Z$. Similarly $h_{2,3} Z$ production also leads to the above final state when $h_{2,3} \rightarrow h_{1} h_{1}$. When $Z$ decays to lepton pair and if we tag only $3 b$ then it can give final state like $3 b+O S D+\left(\left|m_{\ell \ell}-M_{Z}\right| \leq 5 \mathrm{GeV}\right)+\left(\not \not_{\mathrm{T}} \leq 30 \mathrm{GeV}\right)$, where $O S D$ corresponds to opposite sign dilepton.

Comparing the production cross-sections from Table 2 and decay branching fractions from Table 1 and Table 5, we see that the contribution from $h_{1} h_{2,3}$ production would be negligible due to low $h_{2,3} \rightarrow h_{1} Z$ branching fraction for the chosen benchmark points. On the other hand $h_{3} Z$ has relatively large production cross section at least for $\mathrm{BP} 1$ and $\mathrm{BP} 2$ but fails to contribute due to demand of $3 b$ tagging coming from very light Higgs $\left(h_{1}\right)$. One needs to go for very high luminosity to look for this final state. 


\subsection{Sig2: $2 b+2 \tau$}

Unlike for the other benchmark points, in BP3, $h_{3} \rightarrow h_{1} h_{1}$ is very small, and $h_{3}$ mostly decays to $b$ or tau pairs. Thus the final state with $2 b+2 \tau$ looks promising. Thus both the heavy $\left(h_{2,3}\right)$ and light $\left(h_{1}\right)$ Higgs bosons can decay either to $b$ pair or $\tau$ pair which leads to $2 b+2 \tau$ final state. If the $b \mathrm{~s}$ and $\tau \mathrm{s}$ are coming from the heavier Higgs $\left(h_{2,3}\right)$, then they have a high $p_{T}$. On the other hand when they come from the light Higgs $\left(h_{1}\right)$ they have a very low $p_{T}$. We study the final state as:

$$
\operatorname{sig} 2: \mathrm{n}_{\text {jets }} \leq 5+(\geq 2 \mathrm{~b}-\text { jet })+(\geq 2 \tau-\text { jet })+\left(\not p_{\mathrm{T}} \leq 30 \mathrm{GeV}\right) .
$$

Table 7 presents the number of events for the signal and backgrounds at an integrated luminosity of $10 \mathrm{fb}^{-1}$. We can see that sig2 has $13.5 \sigma, 10 \sigma$ and $0.6 \sigma$ significance with 10 $\mathrm{fb}^{-1}$ of luminosity for BP1, BP2 and BP3, respectively. This could be a useful channel to look for the light Higgs scenarios. We then put a window cut in the $b \bar{b}$ invariant mass distribution around the light Higgs mass peak $\left(m_{h_{1}}\right)$ as $\left|m_{b b}-m_{h_{1}}\right| \leq 10 \mathrm{GeV}$. The signal significance for this case does not change much from the previous one and it is $12 \sigma$ and $10.4 \sigma$ for BP1 and BP2. The buried Higgs scenarios can be probed at the LHC. Even when we put the window cut around the next mass peak, i.e., $\left|m_{b b}-m_{h_{2}}\right| \leq 10 \mathrm{GeV}$, the signal significance for BP1 and BP2 still remains around $5 \sigma$ at an integrated luminosity of $10 \mathrm{fb}^{-1}$. For heavier Higgs mass peak resolution, i.e for $m_{h_{3}}$ one needs to go to higher luminosity, at least to $43 \mathrm{fb}^{-1}$ of luminosity in the case of BP1 and BP2. We also investigate the scenario where we take window cuts around $\tau \tau$ invariant mass peak. In this case the reach for the Higgs mass peaks is possible in relatively higher luminosity.

\subsection{Sig3: $2 \ell$}

In this section we will see the exclusive leptonic final states, i.e., the final states with $\mu$ and $e$. Though the branching fractions of Higgses to lepton pair are very small, these tiny branching fractions can be crucial for precision measurement of invariant mass peak. The leptonic channel is particularly handy when it comes to determination of a very small Higgs mass $(\lesssim 50 \mathrm{GeV})$. In Figure 6 the lepton $p_{T}$ distribution comes from (a) $h_{1} h_{3}$ for BP1 and (b) $h_{1} h_{1}$ for BP3. Clearly leptons for BP1 can be treated as hard leptons $\left(p_{T} \geq 20 \mathrm{GeV}\right)$ but for BP3 they can be as hard as $200 \mathrm{GeV}$.

Figure 7 describes the dilepton invariant mass coming (a) from $h_{1} h_{1}$ and (b) from $h_{1} h_{2}$. From Figure ब(a) we can see the $h_{1}$ peaks for all three benchmark points. On the other hand Figure 1(b) shows both the Higgs mass peaks, i.e., $h_{1}$ around 30 and $50 \mathrm{GeV}$, $h_{2}$ around $95 \mathrm{GeV}$.

We first analyse the dilepton final states which could be interesting in determining the very light Higgs scenario with precision. We define the final state as: sig3 : $2 \ell$. Table 8 presents the number of events for the final state sig3 for both signal and backgrounds at an integrated luminosity of $10 \mathrm{fb}^{-1}$. The dominant background events are coming from $t \bar{t}$ and gauge boson pair production $(V V)$. The signal significance for the dilepton final state (sig3) reaches $5 \sigma$ for $\mathrm{BP} 2$ only. The signal significance for $\mathrm{BP} 1$ crosses $3 \sigma$ at $10 \mathrm{fb}^{-1}$ of luminosity. For the light Higgs $\left(h_{1}\right)$ mass peak the significance is $7.6 \sigma$ for BP2 at $10 \mathrm{fb}^{-1}$ 


\begin{tabular}{|c|c|c|c|c|c|c|c|c|}
\hline \multirow[t]{2}{*}{ Signal } & \multicolumn{3}{|c|}{ Benchmark Points } & \multicolumn{5}{|c|}{ Backgrounds } \\
\hline & $\mathrm{BP} 1$ & BP2 & BP3 & $t \bar{t}$ & $t \bar{t} Z$ & $t \bar{t} W$ & $Z Z$ & $t \bar{t} b \bar{b}$ \\
\hline sig2 & 501.30 & 350.80 & 19.00 & 812.10 & 0.30 & 0.50 & 57.70 & 0.20 \\
\hline \multirow{3}{*}{$\operatorname{sig} 2+\left|m_{b b}-m_{h_{1}}\right| \leq 10 \mathrm{GeV}$} & \multirow{3}{*}{195.00} & \multirow{3}{*}{129.00} & \multirow{3}{*}{4.00} & 65.00 & 0.04 & 0.05 & 6.20 & 0.00 \\
\hline & & & & 23.70 & 0.00 & 0.00 & 0.60 & 0.00 \\
\hline & & & & 59.00 & 0.05 & 0.05 & 0.60 & 0.00 \\
\hline \multirow{3}{*}{$\operatorname{sig} 2+\left|m_{b b}-m_{h_{2}}\right| \leq 10 \mathrm{GeV}$} & \multirow{3}{*}{69.00} & \multirow{3}{*}{56.00} & \multirow{3}{*}{0.00} & 103.00 & 0.01 & 0.08 & 15.00 & 0.06 \\
\hline & & & & 104.10 & 0.01 & 0.08 & 16.00 & 0.06 \\
\hline & & & & 1.0 & 0.00 & 0.00 & 0.00 & 0.00 \\
\hline \multirow{3}{*}{$\operatorname{sig} 2+\left|m_{b b}-m_{h_{3}}\right| \leq 10 \mathrm{GeV}$} & \multirow{3}{*}{22.00} & \multirow{3}{*}{8.20} & \multirow{3}{*}{0.00} & 60.00 & 0.04 & 0.06 & 0.30 & 0.00 \\
\hline & & & & 60.00 & 0.04 & 0.06 & 0.30 & 0.00 \\
\hline & & & & 1.00 & 0.00 & 0.00 & 0.00 & 0.00 \\
\hline sig2+|$m_{\tau \tau}-m_{h 1} \mid \leq 10 \mathrm{GeV}$ & 0.10 & 0.00 & 0.00 & 0.00 & 0.00 & 0.00 & 0.00 & 0.00 \\
\hline \multirow{3}{*}{$\operatorname{sig} 2+\left|m_{\tau \tau}-m_{h 2}\right| \leq 10 \mathrm{GeV}$} & \multirow{3}{*}{52.00} & \multirow{3}{*}{33.00} & \multirow{3}{*}{0.20} & 101.00 & 0.04 & 0.10 & 17.00 & 0.06 \\
\hline & & & & 103.00 & 0.04 & 0.10 & 17.00 & 0.06 \\
\hline & & & & 1.00 & 0.00 & 0.00 & 0.00 & 0.00 \\
\hline \multirow{3}{*}{$\operatorname{sig} 2+\left|m_{\tau \tau}-m_{h 3}\right| \leq 10 \mathrm{GeV}$} & \multirow{3}{*}{4.00} & \multirow{3}{*}{3.00} & \multirow{3}{*}{0.10} & 105.00 & 0.01 & 0.07 & 0.30 & 0.06 \\
\hline & & & & 104.00 & 0.03 & 0.07 & 0.20 & 0.00 \\
\hline & & & & 1.00 & 0.00 & 0.00 & 0.00 & 0.00 \\
\hline
\end{tabular}

Table 7: Number of events after the selection cuts for sig2 final states for the benchmark points and backgrounds at an integrated luminosity of $10 \mathrm{fb}^{-1}$ at the LHC with $E_{c m}=14 \mathrm{TeV}$. The different rows of background events for a given column correspond to BP1, BP2 and BP3, respectively as they differ depending on the window cuts around the mass peaks.

of luminosity but for other benchmark points one needs higher luminosity. Specially to determine the Higgs mass peak for BP3 a very high luminosity is needed.

Next we also investigated the $4 \ell$ final state where both the Higgses decay into lepton pairs. The prospect for this final state does not look promising at least for low luminosity and with $14 \mathrm{TeV}$ LHC.

So far we have presented the dominant Standard Model (SM) backgrounds that contribute to the final states. There are other reducible model backgrounds which we also have calculated. They are $H^{ \pm} W^{\mp}, H^{ \pm} h_{i=1,2,3}, H^{ \pm} H^{\mp}$, respectively. We find that their contributions are negligible for the final states we have considered here. The susy backgrounds and supersymmetric backgrounds associated with charged Higgs production have been addressed in great detail in 25] and it is shown that most of the time the final states in supersymmetric cascade decays come with large number of jets, which is unlike the case here.

\section{Summary and discussion}

From our analysis it is clear that the Higgs pair production is interesting in spite of being 


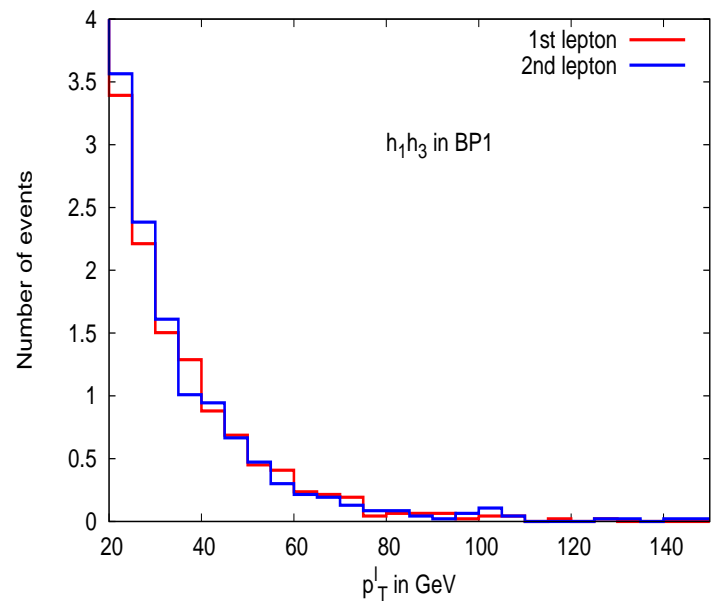

(a)

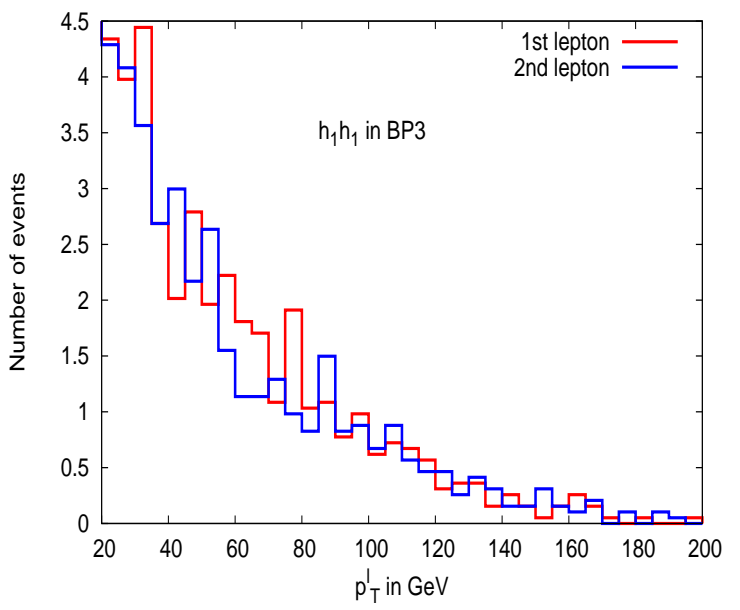

(b)

Figure 6: $p_{T}^{\ell}$ distribution from (a) $h_{1} h_{3}$ for BP1 and from (b) $h_{1} h_{1}$ for BP3.

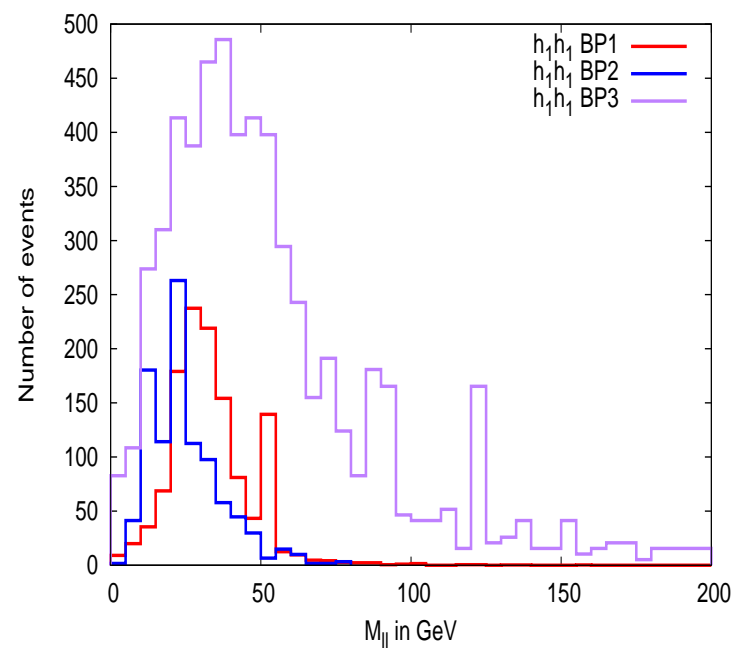

(a)

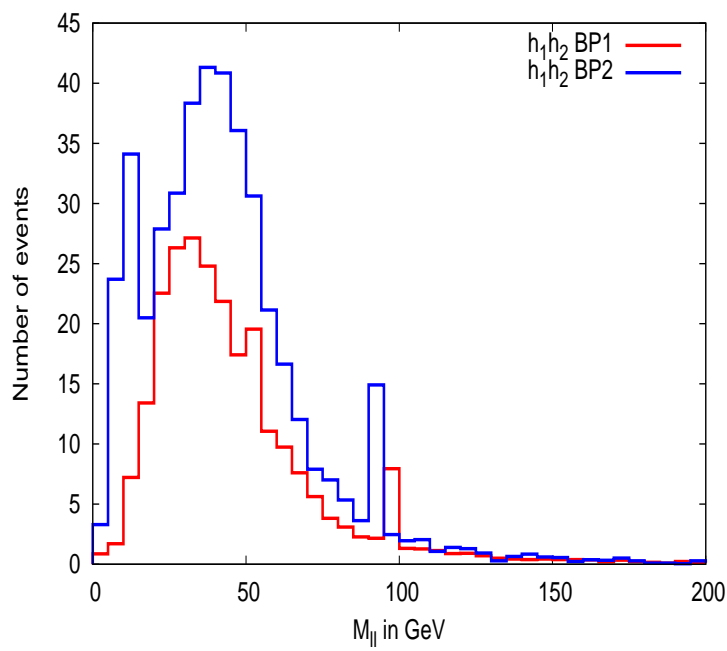

(b)

Figure 7: Lepton invariant mass distribution coming (a) from $h_{1} h_{1}$, (b) from $h_{1} h_{2}$ for benchmark pints at an integrated luminosity of $\mathcal{L}=10 \mathrm{fb}^{-1}$.

electroweak production process. We have studied various possible final states that could come from the two Higgs productions. For some signal topologies an integrated luminosity of $10 \mathrm{fb}^{-1}$ is enough to reach $5 \sigma$ of signal significance. Specially $2 b+2 \tau$ (Sig2) final state looks promising. We have seen that it is also possible to reconstruct the Higgs mass peak, both via $b b$ invariant mass and through $\tau \tau$ invariant mass distribution.

We have also studied the leptonic final state which also has a great prospect due to its precision measurement possibility and can come handy for light Higgs mass discovery. The signal topologies coming from Higgs pair productions are very different from CP-conserving 


\begin{tabular}{|c|c|c|c|c|c|c|c|c|}
\hline \multirow[t]{2}{*}{ Signal } & \multicolumn{3}{|c|}{ Benchmark Points } & \multicolumn{5}{|c|}{ Backgrounds } \\
\hline & $\mathrm{BP} 1$ & $\mathrm{BP} 2$ & BP3 & $t \bar{t}$ & $t \bar{t} Z$ & $t \bar{t} W$ & $V V$ & $t \bar{t} b \bar{b}$ \\
\hline sig3: $2 \ell$ & 200.00 & 370.00 & 15.00 & 2007.00 & 52.00 & 43.00 & 1590.50 & 3.00 \\
\hline \multirow{3}{*}{$\operatorname{sig} 3+\left|m_{\ell \ell}-m_{h_{1}}\right| \leq 10 \mathrm{GeV}$} & \multirow{3}{*}{20.00} & \multirow{3}{*}{205.00} & \multirow{3}{*}{0.60} & 485.00 & 6.00 & 7.80 & 28.00 & 0.40 \\
\hline & & & & 491.00 & 4.00 & 5.00 & 15.00 & 0.70 \\
\hline & & & & 53.00 & 3.60 & 4.20 & 5.00 & 0.10 \\
\hline \multirow{3}{*}{$\operatorname{sig} 3+\left|m_{\ell \ell}-m_{h_{2}}\right| \leq 10 \mathrm{GeV}$} & \multirow{3}{*}{5.00} & \multirow{3}{*}{5.20} & \multirow{3}{*}{0.00} & 153.00 & 5.00 & 6.40 & 706.00 & 0.20 \\
\hline & & & & 156.00 & 5.00 & 6.40 & 710.00 & 0.20 \\
\hline & & & & 0.00 & 0.01 & 0.05 & 1.20 & 0.00 \\
\hline \multirow{3}{*}{$\operatorname{sig} 3+\left|m_{\ell \ell}-m_{h_{3}}\right| \leq 10 \mathrm{GeV}$} & \multirow{3}{*}{0.70} & \multirow{3}{*}{0.50} & \multirow{3}{*}{0.00} & 53.00 & 3.60 & 4.30 & 63.00 & 0.20 \\
\hline & & & & 53.00 & 3.60 & 4.30 & 62.00 & 0.20 \\
\hline & & & & 0.00 & 0.01 & 0.03 & 1.10 & 0.00 \\
\hline
\end{tabular}

Table 8: Number of events after the selection cuts for $2 \ell$ (sig3) final states for the benchmark points and backgrounds at an integrated luminosity of $10 \mathrm{fb}^{-1}$ at the LHC with $E_{c m}=14 \mathrm{TeV}$. The different rows of background events for a given column correspond to BP1, BP2 and BP3, respectively as they differ depending on the window cuts around the mass peaks.

case due to the existence of the light buried Higgs. LHC at $14 \mathrm{TeV}$ has a good chance to explore this possibility once it starts taking data. With more data coming in one can look for $b b \tau \tau$ or $b b \ell \ell$ invariant mass which can determine the heavy Higgs $\left(h_{2,3}\right)$ mass peak and also one can distinguish $h_{i} Z$ events from $h_{i} h_{j}$ events.

\section{Acknowledgments:}

The authors acknowledge support from the Academy of Finland (Project No 137960). PB wants to thank Helsinki Institute of Physics for the visit during the early stages of the project and KIAS overseas travel grant. PB also thanks Prof. Jae Sik Lee for useful discussions.

\section{References}

[1] A. Pilaftsis, Phys. Lett. B 435, 88 (1998) [arXiv:hep-ph/9805373].

[2] A. Pilaftsis and C. E. M. Wagner, Nucl. Phys. B 553, 3 (1999) [arXiv:hep-ph/9902371];

[3] D. A. Demir, Phys. Rev. D 60, 055006 (1999) [arXiv:hep-ph/9901389];

[4] S. Y. Choi, M. Drees and J. S. Lee, Phys. Lett. B 481, 57 (2000) [arXiv:hep-ph/0002287];

[5] M. S. Carena, J. R. Ellis, A. Pilaftsis and C. E. M. Wagner, Nucl. Phys. B 586 (2000) 92 [hep-ph/0003180].

[6] G. L. Kane and L. T. Wang, Phys. Lett. B 488, 383 (2000) [arXiv:hep-ph/0003198];

[7] M. Dugan, B. Grinstein and L. J. Hall, Nucl. Phys. B 255 (1985) 413. S. Dimopoulos and S. D. Thomas, Nucl. Phys. B 465 (1996) 23 [arXiv:hep-ph/9510220]. Y. Kizukuri, N. Oshimo, Phys. Rev. D46, 3025-3033 (1992). 
[8] P. Nath, Phys. Rev. Lett. 66, 2565 (1991); Y. Kizukuri and N. Oshimo, Phys. Rev. D 46, 3025 (1992); T. Ibrahim and P. Nath Phys. Lett. B 418, 98 (1998); Phys. Rev. D 57, 478 (1998); ibid D 58, 019901(E) (1998); ibid D 60, 079903 (1999); ibid D 60, 119901 (1999); M. Brhlik, G.J. Good and G.L. Kane, Phys. Rev. D 59, 115004 (1999); A. Bartl, T. Gajdosik, W. Porod, P. Stockinger and H. Stremnitzer, Phys. Rev. D 60, 073003 (1999); D. Chang, W.-Y. Keung and A. Pilaftsis, Phys. Rev. Lett. 82, 900 (1999); S. Pokorski, J. Rosiek and C.A. Savoy, Nucl. Phys. B 570, 81 (2000); E. Accomando, R. Arnowitt and B. Dutta, Phys. Rev. D 61, 115003 (2000); S. Abel, S. Khalil and O. Lebedev, Nucl. Phys. B 606, 151 (2001); U. Chattopadhyay, T. Ibrahim and D.P. Roy, Phys. Rev. D 64, 013004 (2001); D.A. Demir, M. Pospelov and A. Ritz, hep-ph/0208257.

[9] A. Pilaftsis, Nucl. Phys. B 644, 263 (2002).

[10] T. Falk, K.A. Olive, M. Pospelov and R. Roiban, Nucl. Phys. B 60, 3 (1999).

[11] S. Chatrchyan et al. [CMS Collaboration], Phys. Lett. B 716 (2012) 30 [arXiv:1207.7235 [hep-ex]].

[12] G. Aad et al. [ATLAS Collaboration], Phys. Lett. B 716 (2012) 1 [arXiv:1207.7214 [hep-ex]].

[13] CMS-PAS-HIG-13-001

[14] ATLAS-CONF-2013-012

[15] R. Barate et al. [LEP Working Group for Higgs boson searches], Phys. Lett. B 565, 61 (2003) [arXiv:hep-ex/0306033]; see also http ://lephiggs.web.cern.ch/LEPHIGGS/www/Welcome.html

[16] S. Schael et al. [ALEPH Collaboration], Eur. Phys. J. C 47, 547 (2006) [arXiv:hep-ex/0602042]; see also http : //lephiggs.web.cern.ch/LEPHIGGS/www/Welcome.html G. Abbiendi et al. [OPAL Collaboration], Eur. Phys. J. C 37 (2004) 49 [hep-ex/0406057].

[17] [Tevatron New Physics Higgs Working Group and CDF and D0 Collaborations], arXiv:1207.0449 [hep-ex].

[18] See: LEP Higgs Working Group,LHWG-Note 2004-01.

[19] P. Bechtle [LEP Collaboration], PoS HEP2005, 325 (2006) [arXiv:hep-ex/0602046].

[20] M. S. Carena, J. R. Ellis, A. Pilaftsis and C. E. M. Wagner, Phys. Lett. B 495, 155 (2000) [arXiv:hep-ph/0009212].

[21] A. Pilaftsis, Phys. Rev. D 58, 096010 (1998) [arXiv:hep-ph/9803297].

[22] M. S. Carena, J. R. Ellis, S. Mrenna, A. Pilaftsis and C. E. M. Wagner, Nucl. Phys. B 659, 145 (2003) [arXiv:hep-ph/0211467].

[23] S. P. Das, M. Drees, [arXiv:1010.3701 [hep-ph]]. S. P. Das, M. Drees, [arXiv:1010.2129 [hep-ph]].

[24] E. Accomando et al., [arXiv:hep-ph/0608079].

[25] P. Bandyopadhyay, [arXiv:1008.3339 [hep-ph]].

[26] Z. Li, C. S. Li and Q. Li, Phys. Rev. D 73, 077701 (2006) [arXiv:hep-ph/0601148]; 
[27] P. Bandyopadhyay, A. Datta, A. Datta et al., Phys. Rev. D78 (2008) 015017. [arXiv:0710.3016 [hep-ph]].

[28] P. Bandyopadhyay, JHEP 0907 (2009) 102. [arXiv:0811.2537 [hep-ph]]; P. Bandyopadhyay, A. Datta, B. Mukhopadhyaya, Phys. Lett. B670 (2008) 5-11. [arXiv:0806.2367 [hep-ph]]; K. Huitu, R. Kinnunen, J. Laamanen et al., Eur. Phys. J. C58 (2008) 591-608. [arXiv:0808.3094 [hep-ph]]; A. Datta, A. Djouadi, M. Guchait et al., Nucl. Phys. B681 (2004) 31-64. [hep-ph/0303095]; A. Datta, A. Djouadi, M. Guchait et al., Phys. Rev. D65 (2002) 015007. [hep-ph/0107271]; G. D. Kribs, A. Martin, T. S. Roy and M. Spannowsky, arXiv:1006.1656 [hep-ph]; G. D. Kribs, A. Martin, T. S. Roy and M. Spannowsky, Phys. Rev. D 81 (2010) 111501 [arXiv:0912.4731 [hep-ph]].

[29] D. K. Ghosh, S. Moretti, Eur. Phys. J. C42 (2005) 341-347 [hep-ph/0412365]; D. K. Ghosh, R. M. Godbole, D. P. Roy, Phys. Lett. B628 (2005) 131-140 [hep-ph/0412193];

S. Y. Choi, K. Hagiwara and J. S. Lee, Phys. Rev. D 64, 032004 (2001) [arXiv:hep-ph/0103294]; S. Y. Choi, K. Hagiwara and J. S. Lee, Phys. Lett. B 529, 212 (2002) [arXiv:hep-ph/0110138]; S. Heinemeyer, Eur. Phys. J. C 22, 521 (2001) [arXiv:hep-ph/0108059]; T. Ibrahim and P. Nath, Phys. Rev. D 66, 015005 (2002) [arXiv:hep-ph/0204092]; S. W. Ham, S. K. Oh, E. J. Yoo, C. M. Kim and D. Son, Phys. Rev. D 68, 055003 (2003) [arXiv:hep-ph/0205244]. J. S. Lee, AIP Conf. Proc. 1078 (2009) 36 [arXiv:0808.2014 [hep-ph]].

[30] R. Barate et al. [LEP Working Group for Higgs boson searches and ALEPH Collaboration and and], Phys. Lett. B 565, 61 (2003) [arXiv:hep-ex/0306033]. G. Abbiendi et al. [OPAL Collaboration], Eur. Phys. J. C 37 (2004) 49 [hep-ex/0406057].

[31] J. R. Ellis, J. S. Lee and A. Pilaftsis, JHEP 0810 (2008) 049 [arXiv:0808.1819 [hep-ph]].

[32] K. Cheung, O. C. W. Kong and J. S. Lee, JHEP 0906 (2009) 020 [arXiv:0904.4352 [hep-ph]]. J. Ellis, J. S. Lee and A. Pilaftsis, JHEP 1102 (2011) 045 [arXiv:1101.3529 [hep-ph]].

[33] B. C. Regan, E. D. Commins, C. J. Schmidt and D. DeMille, Phys. Rev. Lett. 88 (2002) 071805 .

[34] P. Franzini, D. Son, P. M. Tuts, S. Youssef, T. Zhao, J. Lee-Franzini, J. Horstkotte and C. Klopfenstein et al., Phys. Rev. D 35 (1987) 2883. J. S. Lee and S. Scopel, Phys. Rev. D 75 (2007) 075001 [hep-ph/0701221 [HEP-PH]].

[35] S. Chatrchyan et al. [CMS Collaboration], arXiv:1307.5025 [hep-ex]. RAaij et al. [LHCb Collaboration], arXiv:1307.5024 [hep-ex]. RAaij et al. [LHCb Collaboration], Phys. Rev. Lett. 110 (2013) 021801 [arXiv:1211.2674 [hep-ex]].

[36] S. L. Glashow, J. Iliopoulos and L. Maiani, Phys. Rev. D 2 (1970) 1285.

[37] S. Chatrchyan et al. [CMS Collaboration], arXiv:1303.2985 [hep-ex]. ATLAS-CONF-2013-047

[38] Y. Amhis et al. [Heavy Flavor Averaging Group Collaboration], arXiv:1207.1158 [hep-ex].

[39] M. S. Carena, D. Garcia, U. Nierste and C. E. M. Wagner, Phys. Lett. B 499 (2001) 141 [hep-ph/0010003]. G. Degrassi, P. Gambino and G. F. Giudice, JHEP 0012 (2000) 009 [hep-ph/0009337].

[40] G. Aad et al. [ ATLAS Collaboration], arXiv:1308.2631 [hep-ex]. G. Aad et al. [ ATLAS Collaboration], arXiv:1308.1841 [hep-ex]. S. Chatrchyan et al. [ The CMS Collaboration], arXiv:1308.1586 [hep-ex]. See also 'http://moriond.in2p3.fr/QCD/2013/MondayMorning/Favareto.pdf' 
[41] ATLAS-CONF-2013-007, CMS-PAS-SUS-13-007

[42] J. S. Lee, M. Carena, J. Ellis, A. Pilaftsis and C. E. M. Wagner, Comput. Phys. Commun. 184 (2013) 1220 [arXiv:1208.2212 [hep-ph]].

J. S. Lee, M. Carena, J. Ellis, A. Pilaftsis and C. E. M. Wagner, Comput. Phys. Commun. 180 (2009) 312 [arXiv:0712.2360 [hep-ph]].

J. S. Lee, A. Pilaftsis, M. S. Carena, S. Y. Choi, M. Drees, J. R. Ellis and C. E. M. Wagner, Comput. Phys. Commun. 156, 283 (2004) [arXiv:hep-ph/0307377]. J. R. Ellis, J. S. Lee and A. Pilaftsis, Mod. Phys. Lett. A 21, 1405 (2006) [arXiv:hep-ph/0605288].

See also 'http://www.hep.man.ac.uk/u/jslee/dist/CPsuperH/v2/tables.2010.Jun.pdf'

[43] T. Hahn, S. Heinemeyer, W. Hollik, H. Rzehak, G. Weiglein and K. Williams, [arXiv:hep-ph/0611373].

[44] [Tevatron Electroweak Working Group and CDF and D0 Collaborations], arXiv:1107.5255 [hep-ex].

[45] A. Pukhov, "CalcHEP 3.2: MSSM, structure functions, event generation, batchs, and generation of matrix elements for other packages", [arXiv:hep-ph/0412191].

[46] See "http://www.personal.soton.ac.uk/ab1u06//public/calchep/"

[47] P. Skands et al., JHEP 0407, 036 (2004) [arXiv:hep-ph/0311123];

see also http://home.fnal.gov/skands/slha/

[48] T. Sjostrand, L. Lonnblad and S. Mrenna, [arXiv:hep-ph/0108264].

[49] H. L. Lai et al. [CTEQ Collaboration], Eur. Phys. J. C 12, 375 (2000) [arXiv:hep-ph/9903282].

[50] J. Pumplin, D. R. Stump, J. Huston, H. L. Lai, P. Nadolsky and W. K. Tung, JHEP 0207, 012 (2002) [arXiv:hep-ph/0201195].

[51] M. Cacciari, G. P. Salam and G. Soyez, Eur. Phys. J. C 72 (2012) 1896 [arXiv:1111.6097 [hep-ph]].

[52] http://www - d0.fnal.gov/ flera/btag ote $_{4} 432 . p d f$

I Tomalin, 2008 J. Phys.: Conf. Ser. 110092033

H. Baer, V. Barger, G. Shaughnessy, H. Summy and L. t. Wang, Phys. Rev. D 75, 095010 (2007) [arXiv:hep-ph/0703289].

[53] G. L. Bayatian et al. [ CMS Collaboration ], J. Phys. G G34 (2007) 995-1579.

[54] G. Bagliesi, [arXiv:0707.0928 [hep-ex]]. 